\title{
Small Bowel Infarction by Mesenteric Venous Thrombosis due to Methylenetetrahydrofolate Reductase Gene Mutation
}

\author{
In Young Park', Byoung Joo Do', Jae Sung Ahn', Jae Hyuk Lee', Jun Ho Park²,3, Jin Gu Kang ${ }^{1,3}$, Bo Kyung Yang ${ }^{1,3}$, Hyoung Su Kim ${ }^{1,3}$ \\ Departments of 'Internal Medicine, ${ }^{2}$ Surgery, and ${ }^{3}$ Digestive Disease Center, Hallym University Kangdong Sacred Heart Hospital, Hallym University College of \\ Medicine, Seoul, Korea
}

\begin{abstract}
Acute mesenteric venous thrombosis (MVT) is an uncommon form of intestinal ischemia with high mortality and usually occurs in the setting of preexisting comorbidities including thrombophilia and abdominal inflammatory conditions. Hyperhomocysteinemia has been known to be a risk factor for thromboembolism, often located on an unusual site. Considering that homocysteine metabolism is determined genetically to a high degree, a mutant of methylenetetrahydrofolate reductase (MTHFR) C677T causes hyperhomocysteinemia, leading to thrombophilia. Until now, there have been few reports of MVT associated with MTHFR gene mutation. We, herein, report a case of small bowel infarction associated with MVT by MTHFR gene mutation in an adult without any other risk factors of thrombophilia.
\end{abstract}

Keywords: Methylenetetrahydrofolate reductase; Hyperhomocysteinemia; Mesenteric veins; Thrombosis; Mutation

\section{INTRODUCTION}

Mesenteric venous thrombosis (MVT) is an uncommon form of mesenteric ischemic events with high mortality. It accounts for $5 \%$ to $15 \%$ of all mesenteric ischemia events, mainly involving superior mesenteric vein (SMV) and usually occurring in a predisposing terrain, whereas the exact causes cannot be identified in about a quarter of patients. The most common causes are hypercoagulable states due to disorders of coagulation, cancer, intraabdominal inflammatory conditions, postoperative state, cirrhosis, and portal hypertension [1]. Recently, hyperhomocysteinemia has been identified to be a risk factor for thromboembolism. Methylenetetrahydrofolate reductase (MTHFR) plays a key role in folate metabolism and in the homeostasis of homocysteine. Mutations in the enzyme subsequently result in an abnormal metabolism, e.g., hyperhomocysteinemia. Until now, it has been reported that C677T polymorphism in MTHFR gene is related to various diseases such as cardiovascular diseases, Alzheimer's disease, depression, some cancers, and neural tube defects [2]. However, there are rare reports of MVT associated with MTHFR gene mutation [3].
We recently experienced a rare case of small bowel infarction associated with MVT by hyperhomocysteinemia due to the C677T mutation in MTHFR gene. Herein, we report the case together with a review of the literature.

\section{CASE REPORT}

A 52-year-old male presented to the emergency department with hematochezia and abdominal pain for 5 days on March, 2012. He had no significant past medical history of vascular disease or chronic medical conditions. He denied personal or family history of thrombosis. He looked vitally stable at first with blood pressure of $120 / 70 \mathrm{~mm} \mathrm{Hg}$, heart rates of 112 beats/min, and body temperature of $37.1^{\circ} \mathrm{C}$. On physical examination, there was mild abdominal tenderness, but not rebound tenderness. The rest of the examination was unremarkable. Initial laboratory findings were as follows: white blood cell 13,640/ $\mathrm{LL}$ (neutrophil $83 \%$ ), hemoglobin $13.8 \mathrm{~g} / \mathrm{dL}$, platelet $22,000 / \mu \mathrm{L}, \mathrm{C}$-reactive protein $72.7 \mathrm{mg} / \mathrm{L}$, total bilirubin $1.2 \mathrm{mg} / \mathrm{dL}$, albumin $3.2 \mathrm{~g} / \mathrm{dL}$, international normalized ratio 1.25 , aspartate transaminase $26 \mathrm{IU} / \mathrm{L}$, alanine transami- 

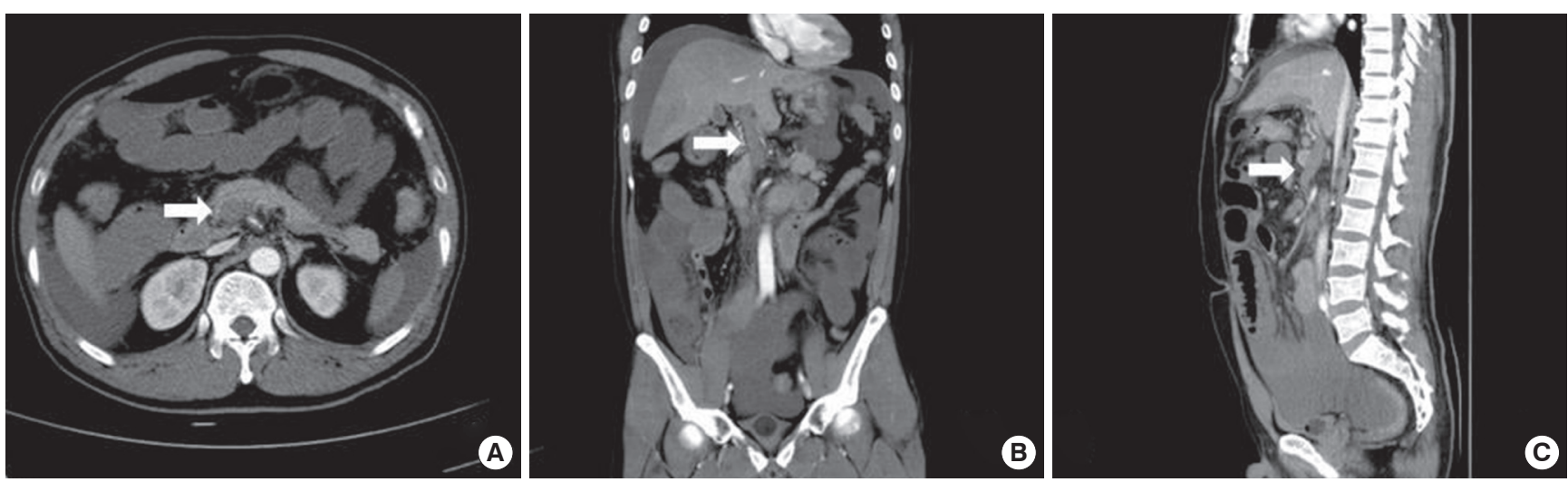

Fig. 1. Abdominal computed tomography showed massive thrombus in portal vein and superior mesenteric vein, ascites and small bowel wall thickening. (A) Transverse view (arrow). (B) Coronal view (arrow). (C) Sagittal view (arrow).

nase $31 \mathrm{IU} / \mathrm{L}$, and serum creatinine $1.9 \mathrm{mg} / \mathrm{dL}$. He was referred to our digestive disease center for evaluation of hematochezia. Esophagogastroduodenoscopy and colonoscopy were performed promptly. Esophagogastroduodenoscopy showed esophageal varix, but no bleeding evidence. On colonoscopy, blood clots were coated on the entire colon and fresh blood was observed inside the ileocecal valve. Under the suspicion of small bowel bleeding, abdominal computed tomography (CT) and angiography were performed. They showed the small bowel wall thickening and ascites with massive thrombosis of portal vein (Fig. 1A, transverse view; 1B, coronal view; 1C, sagittal view), SMV (Fig. 1A), and inferior mesenteric vein but no extravasation of contrast material. This indicated small bowel infarction due to MVT. His abdominal pain was aggravated by degrees and rebound tenderness was accompanied. In eight hours after admission, hemoglobin decreased from $13.8 \mathrm{~g} / \mathrm{dL}$ to $6.1 \mathrm{~g} / \mathrm{dL}$ and blood pressure declined to $80 / 50 \mathrm{~mm} \mathrm{Hg}$. Emergent open laparotomy revealed a necrosis of near total length of small bowel sparing duodenum and a part of ileum. The affected bowel segment was resected (Fig. 2). The postoperative course was favorable. After clinical stabilization, the causes of thrombophilia were screened as for factor $V$ Leiden, factor II G20210A polymorphism, protein C, protein S, antithrombin, antiphospholipid antibodies, vitamin $\mathrm{B}_{12}$, homocysteine, and folate. Of them, only hyperhomocysteinemia $(23.93 \mu \mathrm{mol} / \mathrm{L}$; normal range, 5 to $15 \mu \mathrm{mol} / \mathrm{L})$ and low folate levels $(1.3 \mathrm{ng} / \mathrm{mL}$; normal range, 5.38 to $24 \mathrm{ng} / \mathrm{mL}$ ) were observed. Molecular analysis of the MTHFR gene using restriction fragments length polymorphism exhibited MTHFR C677T homozygous TT genotype. A life-long anticoagulant therapy, therefore, was recommended because he had experienced a life threatening event of mesenteric ischemia and the genetic risk factor for it. He has been treated with folic acid and warfarin simul-

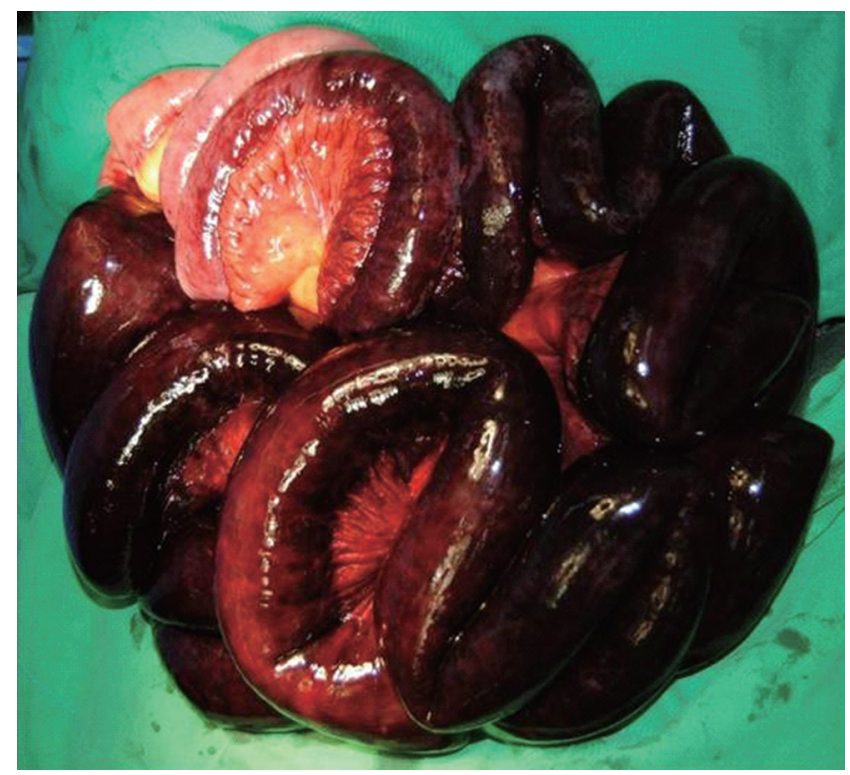

Fig. 2. Necrosis of near total length of small bowel sparing duodenum and a part of ileum was observed, intraoperatively.

taneously. He has been uneventful for 24 months after surgical intervention and medical therapy.

\section{DISCUSSION}

MVT was recognized as a cause of intestinal gangrene more than a century ago [4], and as a cause of intestinal infarction distinct from mesenteric arterial occlusion [5]. MVT is an uncommon form of mesenteric ischemia with high mortality, comprising $5 \%$ to $15 \%$ of all mesenteric ischemia events and usually occurs in predisposing conditions. The inherited causes like hyperhomocysteinemia or factor V Leiden deficiency and the acquired hema- 
Park IY, et al. • Small Bowel Infarction by MVT due to MTHFR Gene Mutation

tological conditions like surgery or intraabdominal infections contribute to an increased risk for MVT.

Hyperhomocysteinemia is well known to cause arterial and venous thromboembolism [6], especially, developed on unusual sites and as a recurrent venous thrombosis [7]. Although, the exact mechanisms underlying the vascular toxic effects of homocysteine are still unclear, it is suggested that homocysteine may accelerate thromboembolic tendency by causing oxidative injury to the endothelium, altering the coagulant properties of the blood, and impairing endothelium-dependent vasomotor regulation. Homocysteine is an intermediate product of methionine metabolism, which is further metabolized via two vitamin B dependent transsulphuration and remethylation pathway. Plasma homocysteine concentrations are determined by either nongenetic or genetic factors. Among the many genetic variations associated with homocysteine concentration such as cystathinine $\beta$-synthase, MTHFR, methionine synthase, and methionine synthase reductase, MTHFR gene mutation is most widely investigated as a risk factor for thrombosis [8]. MTHFR catalyzes the reduction of 5,10-methylenetetrahydofolate to 5 -methyltetrahydrofolate, which is utilized by methionine synthase in the synthesis of methionine from homocysteine. The MTHFR gene is located on chromosome 1 at $1 \mathrm{p} 36.3$, and two common alleles, the C677T (thermolabile) allele and the A1298C allele, have been investigated. A common C677T transition in the MTHFR gene results in a thermolabile variant with specific decreased enzymic activity, and is well established as a genetic determinant of hyperhomocysteinemia. The molecular basis of this thermolability is a missense mutation in exon 4 of the MTHFR gene, a cytosine to thymine substitution at nucleotide 677 , which converts an alanine to a valine codon. This mutation leads to a $30 \%$ decrease in enzyme activity in heterozygotes (CT genotype) and a 60\% decrease in homozygotes (TT genotype), which has been associated with moderately increased homocysteine levels, particularly in the presence of low plasma folate levels. A second polymorphism in the MTHFR gene, associated with decreased enzymic activity but not with thermolability corresponds to an adenosine to cytosine transversion at nucleotide 1298 , in exon 7, leading to a glutamate to alanine substitution in the MTHFR protein. Subjects with the 1,298 CC genotype have reduced enzyme activity, but to a lesser extent than those with the 677TT genotype. Although several studies have shown MTHFR gene mutation increased the risk of thrombosis $[9,10]$, MVT associated with mutant MTHFR gene are scarely reported $[3,10]$. Of note, the patient in the previous report of MVT related to MTHFR mutant had other risk factors such as intraabdominal infection and surgery besides MTHFR mutant. This means that the etiologies or risk factors are interacted multifactorially to induce MVT in clinical settings.

In our case, the patient had only hyperhomocysteinemia and low folate related to MTHFR gene mutation as a risk factor for MVT. To our knowledge, this is a very rare case of MVT associated with MTHFR genetic defect alone.

Important determinants of plasma homocysteine obviously include inadequate concentrations of B vitamins such as folate, vitamin $B_{6}$ and vitamin $B_{12}$ participating in homocysteine metabolism as cofactors or substrates. In fact, plasma concentrations of homocysteine are inversely related to plasma concentrations of folate, vitamin $\mathrm{B}_{6}$ and vitamin $\mathrm{B}_{12}$, and can be decreased by their therapy.

The clinical manifestations of MVT depend on the extent of the thrombus, the size of the affected vessels, and the depth of the bowel wall ischemia. When ischemia is restricted to the mucosa, main symptoms consist of abdominal pain and diarrhea, whereas transmural ischemia leads to necrosis, followed by gastrointestinal bleeding, perforation, and peritonitis. Like our case, hemorrhagic manifestations including hematemesis, melena or hematochezia occurs in about $15 \%$ of patients.

MVT is manifested as acute, subacute, or chronic form. Acute form is diagnosed in patients whose symptoms begin suddenly and associated with bowel infarction, subacute form in those who have abdominal pain for days or weeks without bowel infarction, and chronic form in those who present with complications of portal-vein or splenic-vein thrombosis such as esophageal variceal hemorrhage. Although the patient presented with subacute form, esophageal varix and venous collateral circulation may indicate acute exacerbation of chronic MVT in our case.

Due to the low incidence and vague clinical presentation, a high index of suspicion is required to establish an early diagnosis of MVT. The hallmark of mesenteric ischemia is abdominal pain that is not explained by the physical findings. Currently, early diagnosis of MVT has substantially improved due to advancement in image modalities. Of them, abdominal CT is the test of choice that establishes the diagnosis of MVT in about $90 \%$ of cases and may rule out other causes of abdominal pain. In addition, early diagnosis of MVT facilitates nonoperative management with anticoagulation therapy and helps to prevent serious intestinal occlusion and infarction. Once mesenteric venous thrombosis has been 
confirmed, patients should be screened for hereditary or acquired thrombophilia with tests for protein $\mathrm{C}$ and protein $\mathrm{S}$ deficiencies, factor V Leiden, and other mutations, hyperhomocysteinemia, and paroxysmal nocturnal hemoglobinuria.

In this case, we could not prevent bowel infarction by anticoagulation even after early diagnosis of MVT because the patient deteriorated by rapidly progressing bowel necrosis and gastrointestinal bleeding. However, the patient underwent early surgical intervention followed by a life-long anticoagulation and folic acid replacement which ensure a favorable course without recurrent thrombotic event during the long-term follow-up.

Although hyperhomocysteinemia associated MTHFR genetic mutation is a rare cause of MVT, it should be considered in MVT patients. Also, considering hyperhomocysteinemia, low folate level and homozygous TT mutation in MTHFR gene were the risk factors for MVT in our case, folate therapy combined with anticoagulation might decrease the risk of recurrence.

\section{REFERENCES}

1. Kumar S, Sarr MG, Kamath PS. Mesenteric venous thrombosis. N Engl J
Med 2001;345:1683-8.

2. Trimmer EE. Methylenetetrahydrofolate reductase: biochemical characterization and medical significance. Curr Pharm Des 2013;19:2574-93.

3. Hotoleanu C, Andercou O, Andercou A. Mesenteric venous thrombosis with bowelinfarction and hyperhomocysteinemia due to homozygous methylenetetrahydrofolate reductase C677T genotype. Vasc Endovascular Surg 2008;42:477-81.

4. Elliot JW. II. The operative relief of gangrene of intestine due to occlusion of the mesenteric vessels. Ann Surg 1895;21:9-23.

5. Warren S, Eberhard TP. Mesenteric venous thrombosis. Surg Gynecol Obstet 1935;61:102-21.

6. Den Heijer M, Rosendaal FR, Blom HJ, Gerrits WB, Bos GM. Hyperhomocysteinemia and venous thrombosis: a meta-analysis. Thromb Haemost 1998;80:874-7.

7. Den Heijer M, Blom HJ, Gerrits WB, Rosendaal FR, Haak HL, Wijermans $\mathrm{PW}$, et al. Is hyperhomocysteinaemia a risk factor for recurrent venous thrombosis? Lancet 1995;345:882-5.

8. Castro R, Rivera I, Blom HJ, Jakobs C, Tavares de Almeida I. Homocysteinemetabolism, hyperhomocysteinaemia and vascular disease: an overview. J Inherit Metab Dis 2006;29:3-20.

9. Arruda VR, von Zuben PM, Chiaparini LC, Annichino-Bizzacchi JM, Costa FF. The mutation Ala677-->Val in the methylenetetrahydrofolate reductase gene: a riskfactor for arterial disease and venous thrombosis. Thromb Haemost 1997;77:818-21.

10. He JA, Hu XH, Fan YY, Yang J, Zhang ZS, Liu CW, et al. Hyperhomocysteinaemia, low folate concentrations and methylene tetrahydrofolatereductase C677T mutation in acute mesenteric venous thrombosis. Eur J Vasc Endovasc Surg 2010;39:508-13. 